\title{
Preservação de urediniósporos de Puccinia melanocephala, agente causal de ferrugem em cana-de-açúcar
}

\author{
Ely Oliveira Garcia ${ }^{1 *}$, Marcos Virgílio Casagrande ${ }^{2}$, Alejandro Mário Rago ${ }^{3}$ \& Nelson Sidnei Massola Junior. ${ }^{1}$
}

'Departamento de Entomologia, Fitopatologia e Zoologia Agrícola, Escola Superior de Agricultura “Luiz de Queiroz”, Universidade de São Paulo, CP 09, CEP 13418-900, Piracicaba, SP., fax (19)3434-4839, e-mail: nmassola@esalq.usp.br. ${ }^{2}$ Centro de Tecnologia Canavieira (CTC), Faz. Sto. Antônio S/N, CEP 13400-970, Piracicaba, SP. ${ }^{3}$ INTA - EEA Famaillá, Ruta Prov. 301 km 32 - C.C. 9 - CP 4132 - Famaillá, Tucumán, Argentina. *Bolsista FAPESP (04/07990-7).

Autor para correspondência: Nelson S. Massola Jr.

Data de chegada: 14/10/2005. Aceito para publicação em: 24/10/2006.

\section{RESUMO}

Garcia, E.O; Casagrande, M.V.; Rago, A.M.; Massola Jr., N.S. Preservação de urediniósporos de Puccinia melanocephala, agente causal de ferrugem em cana-de-açúcar. Summa Phytopathologica, v.33, n.2, p.152-156, 2007.

A sazonalidade na manifestação da ferrugem da cana dificulta a obtenção de esporos em quantidades adequadas para inoculações em qualquer época do ano, restringindo os trabalhos envolvendo o patógeno aos meses nos quais a doença esta presente no campo. O trabalho visou desenvolver uma metodologia para preservar os esporos por períodos prolongados, mantendo sua viabilidade e infectividade. Esporos foram coletados a partir de folhas naturalmente infectadas, com bomba de vácuo. Parte dos esporos foi desidratada por liofilização ou em sílica gel e outra parte não passou por desidratação. Armazenaram-se estes esporos em diferentes temperaturas (temp. ambiente, $5^{\circ} \mathrm{C},-20{ }^{\circ} \mathrm{C},-80^{\circ} \mathrm{C}$ ). Periodicamente, a viabilidade dos esporos foi avaliada por meio de plaqueamento em ágar-água. Após o quarto mês, foi também avaliada a infectividade dos esporos armazenados por meio de inoculações na variedade suscetível SP701143, seguida da avaliação da área foliar atacada. Os esporos armazenados à temperatura ambiente e a $5{ }^{\circ} \mathrm{C}$, independentemente da desidratação, permaneceram viáveis por períodos máximos de 1 mês e 2 meses, respectivamente. Os melhores tratamentos consistiram na desidratação em sílica gel, seguida pelo armazenamento à $-20^{\circ} \mathrm{C}$ e $-80^{\circ} \mathrm{C}$. Mesmo após um ano de armazenamento nestas condições, os esporos provocaram ferrugem nas plantas inoculadas, em níveis de severidade adequados para um teste de discriminação de reações à ferrugem.

Palavras-chave adicionais: viabilidade, infectividade, esporos, uredinales.

\section{ABSTRACT}

Garcia, E.O; Casagrande, M.V.; Rago, A.M.; Massola Jr., N.S. Preservation of uredospores of Puccinia melanocephala, the causal agent of sugarcane rust. Summa Phytopathologica, v.33, n.2, p.152-156, 2007.

The seasonality in the appearance of sugarcane rust makes it difficult to obtain in any season of year, adequate quantities uredospores for artificial inoculations in a breeding program. This research aimed to develop a long-term storage methodology to preserve uredospores of Puccicinia melanocephala to keep their viability and infectivity. Uredospores were collect using a vacuum pump from naturally rusted leaves. Part of these uredospores was dehydrated in silica gel or lyophilized and the other part was not dehydrated. Then they were all stored at different temperatures (room temperature, $5^{\circ} \mathrm{C},-20^{\circ} \mathrm{C}$ and $-80^{\circ} \mathrm{C}$ ). Subsequently, uredospores viability was periodically evaluated using the water-agar method. After the fourth month, the infectivity was also evaluated through inoculations in leaves of the sugarcane susceptible variety SP70-1143 following disease evaluation. Regardless of the dehydration treatment used, uredospores stored at room temperature and at $5^{\circ} \mathrm{C}$ were viable for the maximum periods of 1 and 2 months, respectively. The best results were obtained with the silica gel dehydration treatment followed by storage at $-20^{\circ} \mathrm{C}$ or $-80^{\circ} \mathrm{C}$. Even after one year of storage, these uredospores were able to cause rust in inoculated plants at levels of severity which are adequate to start screening tests for sugarcane resistance to rust.

Additional keywords: viability, infectivity, spores, uredinales.

A ferrugem da cana é uma doença relativamente recente no Brasil. Foi relatada no município de Capivari, Estado de São Paulo, no ano de 1986 (7). Esta doença possui elevado potencial destrutivo, e é responsável pelo abandono de variedades comerciais $(6,30,31)$.

A doença é causada pelo basidiomiceto Puccinia melanocephala, parasita obrigatório de tecidos foliares da cultura da cana-de-açúcar (31). As folhas atacadas exibem pústulas salientes, sintomas típicos de algumas ferrugens, com rompimento da epiderme. Estas pústulas apresentam coloração amarelada a marrom-escuro, em função da massa de urediniósporos $(22,31)$. A presença das pústulas reduz a atividade fotossintética resultando na redução do crescimento e queda de produção. Os prejuízos são causados pela redução do desenvolvimento dos colmos, em conseqüência da alta infestação da doença nos primeiros meses de desenvolvimento da cultura (10).

Como a ferrugem é uma doença que apresenta sazonalidade na manifestação de sintomas, com picos de incidências nos meses de 
junho a agosto e de novembro a janeiro, a falta de uma metodologia eficiente de preservação do patógeno restringe as pesquisas sobre o controle da doença a esses meses, impondo restrições aos programas de melhoramento da cana-de-açúcar.

A seleção de métodos adequados à preservação não constitui um assunto recente (14). A literatura especializada é rica na descrição de procedimentos aplicáveis a diferentes gêneros e espécies $(16,27)$. Cada método apresenta vantagens e desvantagens, as quais devem ser consideradas, levando-se em conta as características que se deseja preservar, destacando-se não apenas a manutenção de altos níveis de viabilidade celular, como características morfofisiológicas, taxonômicas e patogênicas, além da prevenção de contaminações indesejáveis (17).

Entre os diversos métodos de preservação de esporos fúngicos disponíveis, deve-se procurar utilizar aqueles que mantenham inalteradas o maior número possível das características dos microrganismos. Isso é particularmente importante no caso dos patógenos de plantas, para os quais a patogenicidade é uma característica essencial $(21,26)$. No caso de patógenos causadores de ferrugens, os métodos de preservação comumente utilizados envolvem liofilização e manutenção em baixas temperaturas (ultrafreezer ou nitrogênio líquido), com boa manutenção de sua viabilidade e patogenicidade $(3,13,23,24)$. Entretanto, pouco foi testado destas metodologias de preservação especificamente para $P$. melanocephala. A preservação dos esporos em condições ambientes ou em geladeira tem se mostrado inadequada, devido à rápida perda da viabilidade, $\mathrm{o}$ que os torna impróprios para as inoculações artificiais.

Dessa forma, o presente trabalho visou desenvolver uma metodologia para preservar os urediniósporos de $P$. melanocephala por períodos relativamente prolongados, mantendo suas características de patogenicidade.

\section{MATERIAL E MÉTODOS}

Seguindo a metodologia proposta por Autrey et al. (3), coletaramse esporos com bomba de vácuo a partir de cultivares suscetíveis de cana naturalmente infectados, obtidos na área experimental do Centro de Tecnologia Canavieira, Piracicaba, SP. Esta coleta foi realizada no mês de julho de 2004 .

Parte destes esporos foi desidratada em sílica gel (48 horas em dessecador a $5^{\circ} \mathrm{C}$ ) ou liofilizada ( 48 horas em liofilizador a $-20^{\circ} \mathrm{C}$ ) e parte permaneceu sem passar por estes processos de desidratação. Os tratamentos consistiram em armazenar esses esporos em cápsulas sob diferentes temperaturas (temp. ambiente; $5^{\circ} \mathrm{C} ;-20{ }^{\circ} \mathrm{C} ;-80^{\circ} \mathrm{C}$ ). Para isto, as cápsulas passaram por gradativa diminuição de temperatura antes de serem armazenadas nas temperaturas de $-20^{\circ} \mathrm{C}$ e $-80^{\circ} \mathrm{C}$, seguindo as recomendações propostas por Costa \& Ferreira (8) e Silva (25). Os esporos liofilizados foram armazenados em temperatura ambiente. Para cada tratamento foram preparadas 15 cápsulas com esporos, as quais foram hermeticamente fechadas.

Avaliou-se a viabilidade dos esporos inicialmente, antes de submetêlos aos tratamentos e, após o início da preservação, quinzenalmente durante os 2 primeiros meses. Após os 2 primeiros meses as avaliações foram mensais, até que fosse completado 1 ano de armazenamento. As avaliações de viabilidade foram realizadas por meio do plaqueamento dos esporos em meio ágar-água (2). Para isso, os esporos foram rehidratados lentamente, deixando-se a cápsula aberta por 4 horas em câmara úmida. Em seguida preparou-se suspensão de esporos $\left(2 \times 10^{5}\right.$ esporos $/ \mathrm{mL}$ ) em água estéril e plaqueou-se uma alíquota de $0,1 \mathrm{~mL}$ sobre o meio água-ágar (1,5\%). Para cada tratamento foram feitas 5 repetições. Após 6 horas de incubação a $21^{\circ} \mathrm{C}$, a germinação foi interrompida por meio da adição de gotas de lactofenol, com posterior deposição de lamínulas na superfície do meio (5). Com o auxílio de um microscópio óptico, usando o aumento de 100 vezes, foram contados o número de esporos germinados ou não, totalizando 200 esporos por repetição. Os resultados foram expressos em porcentagem de germinação.

Após o $4^{\circ}$ mês de armazenamento, paralelamente à avaliação de viabilidade, periodicamente, no mesmo dia de abertura de cada cápsula, foi feita a inoculação destes esporos sobre plantas com 30 dias da variedade suscetível SP70-1143. A inoculação consistiu na aplicação da mesma suspensão de esporos $\left(2 \times 10^{5}\right.$ esporos $\left./ \mathrm{mL}\right)$ sobre a face abaxial da folha +1 (primeira folha que apresenta lígula visível) de cada planta. Em seguida, as plantas permaneceram por 2 dias em câmara úmida e por mais 13 dias em incubadora $\left(21^{\circ} \mathrm{C}\right.$; 12 horas de fotoperíodo). Após este período, procedeu-se à avaliação por meio da escala diagramática proposta por Amorim et al., (1). Utilizaram-se 10 repetições para cada tratamento de preservação.

Para fins de confirmação da eficiência de alguns tratamentos selecionados, o ensaio foi repetido a partir de uma nova coleta de esporos, realizada no mês de fevereiro de 2005. Estes esporos foram submetidos à desidratação e armazenados durante 4 meses a $-20^{\circ} \mathrm{C} \mathrm{e}$ $80^{\circ} \mathrm{C}$, tratamentos considerados promissores durante o primeiro ensaio. As avaliações de viabilidade seguiram a mesma metodologia, porém, a infectividade foi avaliada por meio da inoculação em 3 variedades de cana (SP70-1143 - suscetível, SP91-1397 - intermediária e R570 resistente).

Em algumas das inoculações, amostras de folhas inoculadas foram retiradas e processadas para microscopia eletrônica de varredura (M.E.V.), segundo metodologia de Bozzola \& Russel (4), com objetivo de confirmar a germinação e formação de apressório pelos esporos preservados.

\section{RESULTADOS E DISCUSSÃO}

A análise da viabilidade dos urediniósporos no decorrer do tempo de preservação (figura 1) revelou que o armazenamento nas temperaturas $-20^{\circ} \mathrm{C}$ e $-80^{\circ} \mathrm{C}$ foi o fator preponderante para manter a viabilidade. Os esporos armazenados à temperatura ambiente e a $5^{\circ} \mathrm{C}$ não permaneceram viáveis por mais que 60 dias, independente da desidratação. Os esporos liofilizados também perderam a viabilidade em curto intervalo de tempo, indicando que esse tratamento não foi adequado para preservar $P$. melanocephala, ao contrário do que relataram Sharp \& Smith (23) para P.coronata e P.graminis. Para aqueles armazenados à $-20^{\circ} \mathrm{C}$ observou-se evidente aumento na porcentagem

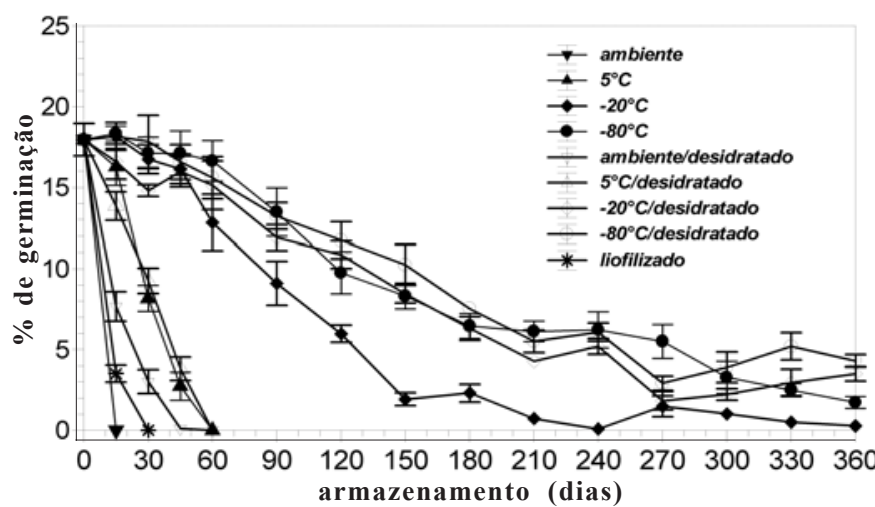

Figura 1. Viabilidade de urediniósporos de Puccinia melanocephala, preservados sob 9 condições, ao longo de um ano. 
de urediniósporos viáveis quando se realizou desidratação prévia. Os tratamentos $-80^{\circ} \mathrm{C}$ sem desidratação e $-80^{\circ} \mathrm{C}$ com desidratação apresentaram eficiências semelhantes.

Os resultados dos testes de infectividade com os esporos armazenados não mostraram correlação com a viabilidade dos mesmos. Apesar disto, foi possível observar que, mesmo após 12 meses de preservação, os tratamentos $-20^{\circ} \mathrm{C}$ com desidratação e $-80^{\circ} \mathrm{C}$ com e sem desidratação mantiveram os esporos infectivos em níveis suficientes para causar boa severidade de ferrugem nas plantas inoculadas. $\mathrm{O}$ armazenamento de esporos não desidratados à $-20^{\circ} \mathrm{C}$ se mostrou inadequado para manter a infectividade dos mesmos. Para este tratamento a severidade de ferrugem foi sempre baixa (figuras $2 \mathrm{e}$ 3). Autrey et al (3) também verificaram, por meio da inoculação em plantas com 90 dias, que esporos de uma população de $P$. melanocephala das Ilhas Maurício, desidratados e armazenados a -

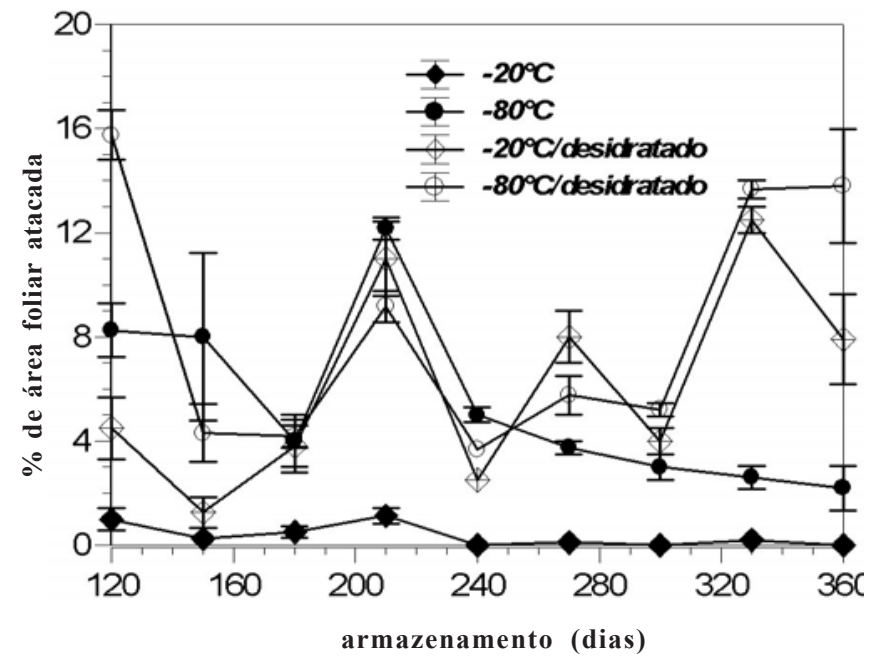

Figura 2. Porcentagem de área foliar atacada pela ferrugem em folhas da variedade suscetível SP70-1143, inoculadas com esporos preservados em 4 condições.

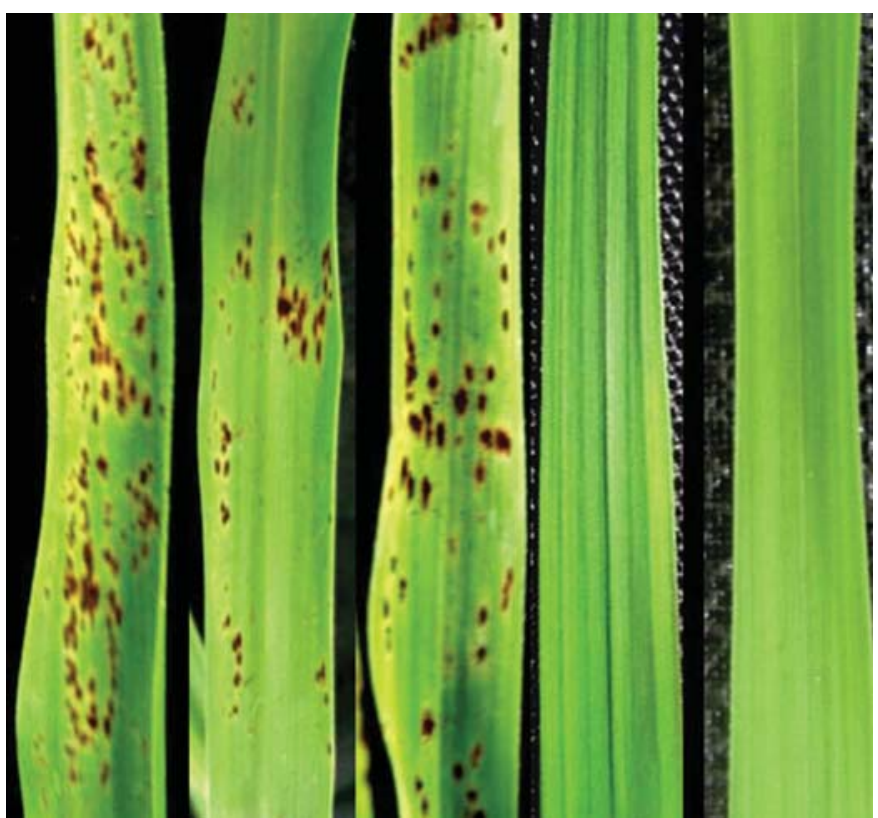

Figura 3. Sintomas de ferrugem em folhas de cana-de-açúcar da variedade suscetível SP70-1143 inoculadas com urediniósporos armazenados durante 12 meses. Da esquerda para direita: tratamentos $-80^{\circ} \mathrm{C} / \mathrm{des}^{*}$., $-80^{\circ} \mathrm{C}$, $20^{\circ} \mathrm{C} /$ des., $-20^{\circ} \mathrm{C}$ e o controle. (des $=$ esporos previamente desidratados). $23^{\circ} \mathrm{C}$ tiveram sua infectividade preservada. Discordando destes resultados, Faleiro et al. (11) relataram drástica redução na capacidade de infecção de urediniósporos de Uromyces appendiculatus após armazenamento em baixas temperaturas. No entanto, estes autores não submeteram os urediniósporos à desidratação antes do armazenamento. Esta constatação sugere que a desidratação prévia é um importante fator para preservar a capacidade infectiva dos urediniósporos.

No segundo ensaio, realizado com os esporos coletados em fevereiro de 2005, novamente comprovou a boa eficiência na preservação de esporos desidratados e armazenados à $-20^{\circ} \mathrm{C} \mathrm{e}-80^{\circ} \mathrm{C}$. Com relação à viabilidade, estes dois tratamentos não apresentaram diferença significativas entre si (figura 4), mantendo bom nível de germinação dos urediniósporos durante os 4 meses do ensaio. A infectividade destes esporos foi também preservada (figura 5), sem diferença significativa entre ambas as temperaturas de armazenamento. A inoculação destes esporos nas 3 variedades de cana permitiu diferenciar a variedade SP70-1143 (suscetível) das SP91-1397 (intermediária) e R570 (resistente), sugerindo a possibilidade do emprego de esporos armazenados nestas condições para testes de reação de genótipos.

A análise em M.E.V. revelou que os esporos preservados apresentam germinação e formação de apressório sobre a superfície das folhas inoculadas (figura 6).

Concluindo, pode-se afirmar que a desidratação seguida do armazenamento em baixas temperaturas foi o método mais eficiente para preservar urediniósporos de P. melanocephala. Tanto os esporos coletados em julho de 2004, com 17,95\% de viabilidade inicial, quanto aqueles coletados em fevereiro de 2005 , com 5,35\% de viabilidade, foram preservados eficientemente dessa maneira. É importante salientar que o armazenamento de esporos desidratados nas temperaturas de $20^{\circ} \mathrm{C}$ e $-80^{\circ} \mathrm{C}$, em ambos os ensaios, apresentaram eficiências muito semelhantes. Portanto, como o armazenamento à $-20^{\circ} \mathrm{C}$ não requer equipamento de valor elevado, sugere-se que este procedimento seja adotado nos laboratórios de instituições que trabalham com a ferrugem de cana-de-açúcar, o que permitirá estudos em épocas do ano que apresentam escassez de urediniósporos em condições naturais.

Apesar do sucesso na preservação aqui relatado, há ainda que se considerar a baixa porcentagem de germinação dos urediniósporos de P. melanocephala encontrada neste trabalho, fato evidenciado também

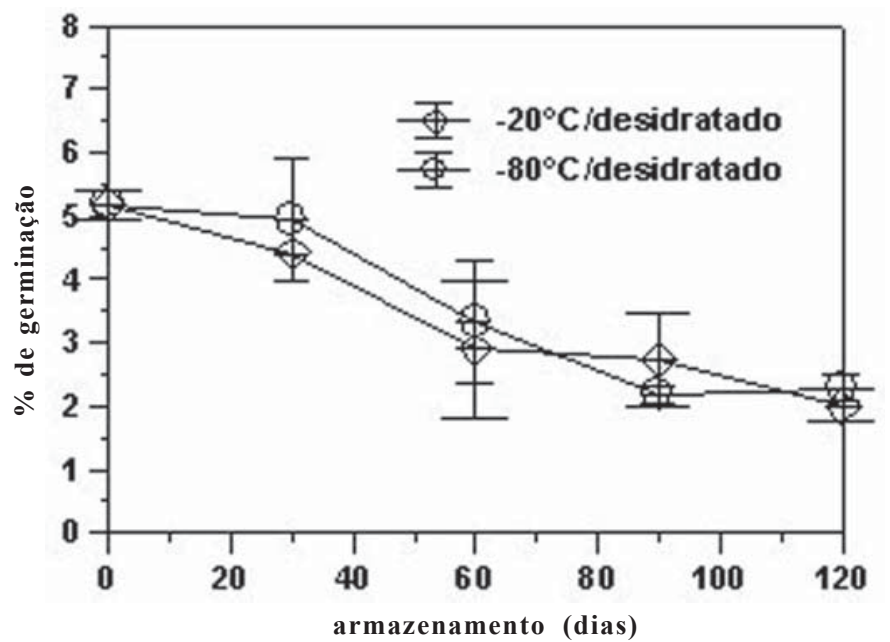

Figura 4. Germinação de urediniósporos de Puccinia melanocephala, preservados sob 2 condições, ao longo de 4 meses. 

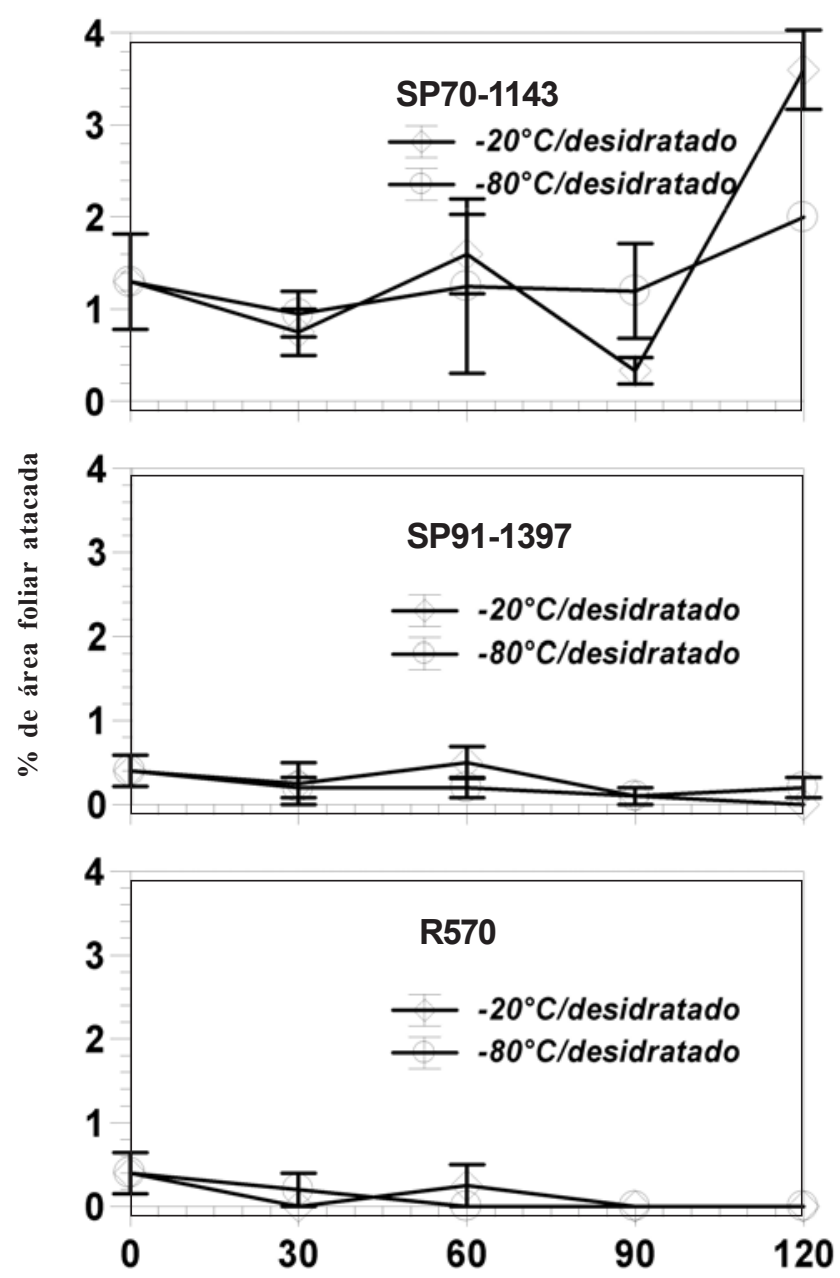

armazenamento (dias)

Figura 5. Porcentagem de área foliar atacada pela ferrugem em folhas de 3 variedades de cana, inoculadas com esporos armazenados em 2 condições.

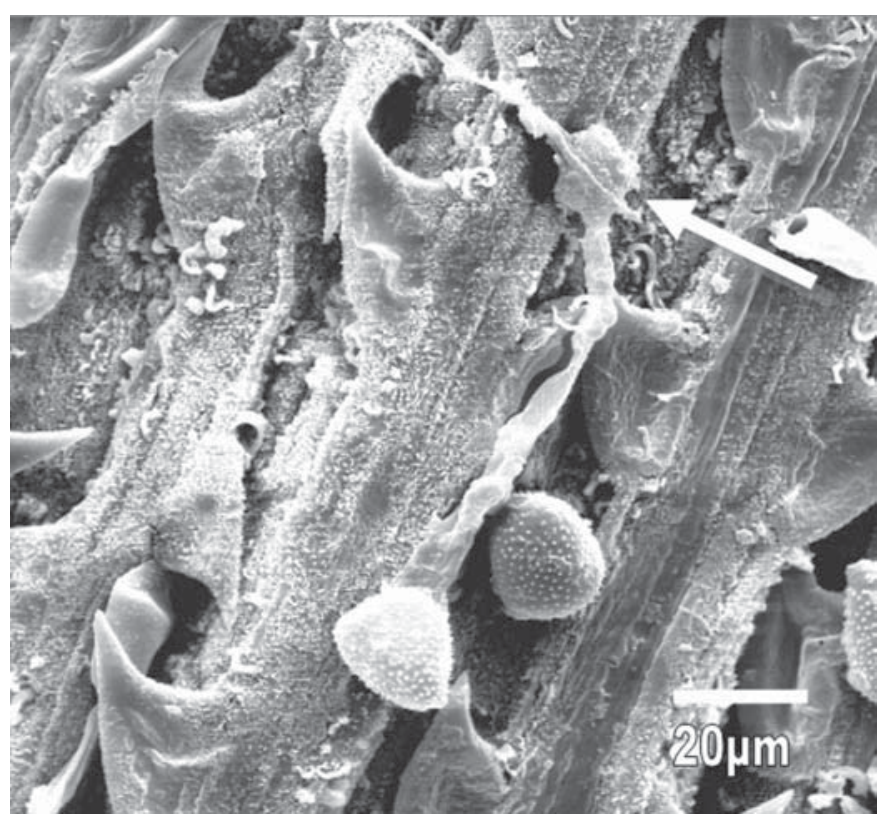

Figura 6. Detalhes do tubo germinativo e do apressório (seta) de urediniósporo preservado por 12 meses, formados sobre folha de canade-açúcar da variedade SP 70-1143 e observados ao microscópio eletrônico de varredura. por outros autores $(15,20)$. Entretanto, Stomayor et al. (29) revelaram que a germinação dos urediniósporos de $P$. melanocephala sobre o tecido foliar da cana é duas vezes maior, em relação ao meio de cultura Ágar-Água. Outros agentes de ferrugem apresentam taxas de germinação mais altas, como, por exemplo, Puccinia psidii e Hemileia vastatrix, entre outros $(9,12,28)$. Este fato pode estar relacionado com a presença de auto-inibidores da germinação nos esporos, como dimetoxicinamatos. Esporos com tais auto-inibidores são capazes de germinar somente após a remoção parcial do composto. Este fato representa uma vantagem ecológica para o fungo, pois previne a germinação dos urediniósporos ao mesmo tempo (28). Dessa forma, se fazem necessários estudos sobre auto-inibição na germinação dos urediniósporos de $P$. melanocephala, visando melhorar a eficiência das inoculações controladas nos ensaios com esse fungo.

\section{REFERÊNCIAS BIBLIOGRÁFICAS}

1. Amorim, L.; Bergamin Filho, A.; Sanguino, A.; Cardoso, C.O.N.; Moraes, V.A.; Fernandes, C. R. Metodologia de avaliação de ferrugem da cana-de-açúcar (Puccinia melanocephala). Boletim Técnico Copersucar, São Paulo, n.39, p.13-16, 1987.

2. Asnaghi, C.; D'hont, A.; Glaszmann, J.C.; Rott, P. Resistance of sugarcane cultivar R570 to Puccinia Melanocephala isolates from different geographic locations. Plant Disease, Saint Paul, v.85, n.3, p.443-450, 2001.

3. Autrey, L.J.C.; Moutia, Y.; Saumtally, S. Screening of seedlings against rust, inoculation treatment and assessment by video image analysis. Sugar Cane, Port Talbot, v.1, p.4-10, 1996.

4. Bozzola, J.J; Russell, L.D. Electron microscopy. 2. ed. Sudbury: Jones and Bartelett Publishers, 1999. 670p.

5. Cardoso, C.O.N.; Fernandes, R.G.; Amorim, L.; Casagrande, M.V.; Moraes, V.A. Etiologia do carvão e da ferrugem da cana-de-açúcar Efeito da temperatura na germinação dos esporos. Boletim Técnico Copersucar, São Paulo, n. 43 p.13-18, jul. 1988.

6. Comstok, J.C.; Wu, K.K.; Schnell, R.J. Heritability of resistance to sugarcane rust. Sugar Cane, Port Talbot, v.6, p.7-10, 1992.

7. Cooperativa Central dos Produtores de Açúcar e Álcool do Estado de São Paulo. Ferrugem da cana-de-açúcar e sua constatação no município de Capivari. Boletim Técnico Copersucar, São Paulo, p.1-8, 1986. Edição especial.

8. Costa, C.P.; Ferreira, M. C. Preservação de microorganismos: revisão. Revista de Microbiologia, São Paulo, v.22, n.3, p.206-208, 1991.

9. Coutinho, T.A.; Wingfield, M. J.; Alfenas, A. C.; Crous, P. W. Eucalyptus Rust: A Disease with the Potential for Serious International Implications. Plant Disease, v.82, n.7, p. 819-825, 1998.

10. Dias, D.S. Um método de avaliação do nível de ferrugem por meio de imagens. STAB, Açúcar, Álcool e Subprodutos, São Paulo, v.23, n.1, p.38-40, 2004.

11. Faleiro, F.G.; Ragamin, V.A.; Vinhadelli, W.S.; Mesquita, A.G.G.; Moreira, M.A.; Barros, E.G. Redução da capacidade de infecção de urediniósporos de Uromyces appendiculatus após o armazenamento em diferentes condições. Fitopatologia Brasileira, Brasília, v.25, n.1, p.98-100, 2000.

12. Garçon, C.L.P.; Zambolin, L.; Mizubuti, E.S.G.; Do Vale, F.X.R.; Costa, H.; Chaves, G.M. Progresso da ferrugem do cafeeiro (Hemileia vastatrix) em diferentes altitudes. Summa Phytopathologica, v.28, n.3, p.267-270, 2002.

13. Green, G.J. Identification of physiologic races of Puccinia graminis f. sp. tritici in Canada. Canadian Journal of Plant Pathology, Ontario, v.3, p.33-39, 1981.

14. Greene, H.C.; Fred, E.B. Maintenance of vigorous mould stock cultures. Industrial Engineering Chemistry, Washington, v.26, p.1297-1299, 1934.

15. Hsueh, W.H. Rust disease of sugar cane in Taiwan: The causal organismo Puccinia melanocephala Syd., Taywan Sugar. v.5, n.3, p.416-419, 1977. 
16. Kirsop, B.E.; Snell, J.J. (Ed.). Maintenance of microorganism: a manual of laboratory methods. London: Academic Press, 1984. 207p.

17. Lopes-Shikida, S.A.R. Isolamento, seleção e preservação de fungos produtores de pectinases. 1995. 122f. Dissertação (Mestrado em Genética) - Escola Superior de Agricultura "Luiz de Queiroz", Universidade de São Paulo, Piracicaba.

18. Ouf, M.F.; Gazar A.A.; Abdou, E.S, Kiraly, Z.; Barna, B. The effect of superoxide anion on germination and infectivity of wheat-stem rust (Puccinia-graminis pers $\mathrm{f} \mathrm{sp}$ tritici-eriks and henn) uredospores. Cereal research communications, Szeged, Hungary, v.21, n.1, p.31-37, 1993 .

19. Parlevliet, J. E., Components of resistance that reduce the rate of epidemic development. Annual Review of Phytopathology. Palo Alto, v.17, p.203-222, 1979.

20. Peros, J.P. Infection de la cane à sucre cultivée in vitro par Puccinia melanocephala Syd. L'agronomie Tropicale, Paris, v.34, n.4, p.355-357, 1984

21. Picinini, E.; Pascholati, S.F. Crescimento, esporulação e patogenicidade de isolados de Colletotrichum graminicola após 14 anos de preservação pelo método de Castellani. Fitopatologia Brasileira, Brasília, v.21, n.4, p.499-502, 1996.

22. Purdy, L.H.; Liu, L.J.; Dean, J.L. Sugarcane rust, a newly important disease. Plant Disease, Saint Paul, v.67, n. 11, p. $1292-1296,1983$

23. Sharp, E.L.; Smith, F.G. Preservation of Puccinia uredospores by lyophilization. Phytopathology, Lancaster, v.42, n.5, p. 263-264, 1952 .
24. Sharp, E.L.; Smith, F.G. Further study of the preservation of Puccinia uredospores. Phytopathology, Lancaster, v.47, n.7, p.423-429, 1957.

25. Silva, L.F. Comparação de métodos de preservação de leveduras de interesse industrial. 1990. 152f. Dissertação (Mestrado em Microbiologia) - Instituto de Ciências Biomédicas, Universidade de São Paulo. São Paulo.

26. Smith, D.; Waller, J.M. Culture colections of microrganisms: their importance in tropical plant pathology. Fitopatologia Brasileira, Brasília, v.17, n.1, p.5-12, 1992.

27. Smith, D.; Onions, A.H.S. The preservation and maintenance of living fungi. Surrey: Commonwealth Mycological Institute, 1983. $51 \mathrm{p}$.

28. Staples, R.C.; Macko, V. Germination of uredospores and differentiation of infection structures. In: Bushnell, W.R.; Roelfs, A.P. The cereal rusts, Saint Paul: Academic Press, 1984. v.1, cap.9, p.255289.

29. Stomayor, I.A.; Purdy, L.M.; Trese, A.T. Infection of sugarcane leaves by Puccinia melanocephala. Phytopathology. v.73, n. 5, p. 695-699, 1983.

30. Taylor, P.W.J. Identification of physiologic single races of common rust caused by Puccinia melanocephala, in Australian sugar cane cultivars. Australian Journal of Agricultural Research, Melbourne, v.43, p.443-450, 1992.

31. Tokeshi, H; Rago, A. Doenças da cana-de-açúcar. In: Kimati, H.; Amorim, L.; Rezende, J.A.M.; Bergamin Filho, A.; Camargo, L.E.A. Manual de fitopatologia: doenças das plantas cultivadas. São Paulo: Agronômica Ceres, 2005. v.2, cap. 21, p.185-196. 